\title{
The Development of Tourism Industry Has Studied the Promotion Effect of Accurate Poverty Alleviation in Guizhou County
}

\author{
Siqi Li ${ }^{1, a}$ Mu Zhang ${ }^{1, b}$
}

School of Finance, Guizhou University of Finance and Economics, Guiyang Guizhou 550025, China a546932209@qq.com, brim_007@163.com

\begin{abstract}
In this paper, the author of this paper is how to translate the advantages of natural resources and national cultural resources into economic advantages. In this paper, the tourism industry of 50 poverty-stricken counties in Guizhou province is selected as the representative, and then the antipoverty effect of Guizhou tourism industry is measured by using panel data model. The empirical results show that the 50 counties in Guizhou tourism development will promote the development of national economy, thereby reducing the incidence of poverty counties in Guizhou, and concluded that the tourism industry in Guizhou in Guizhou county precision poverty alleviation poverty alleviation has a certain role in promoting.
\end{abstract}

\section{Keywords}

Tourism Industry, Guizhou County, Targeted Poverty Alleviation, Panel Data Model.

\section{旅游产业发展对贵州县域精准扶贫的促 进作用研究}

\author{
李思锜 $1, \mathrm{a}$ 张目 $1, \mathrm{~b}$ \\ 1 贵州财经大学金融学院,花溪,贵阳,贵州 550025,中国 \\ 2249086572@qq.com, brim_007@163.com
}

摘要：贵州县域交通闭塞、经济区位偏远，如何把自身所具有的特色自然资源、民族文化资 源等优势转换为经济优势, 这是本文的立文之本。本文选取了贵州省 50 个贫困县的旅游产 业作为代表, 然后运用面板数据模型对贵州旅游产业的扶贫效应进行测度。实证结果表明, 贵州这 50 个贫困县旅游产业的发展促进了国民经济的发展，从而降低了贵州各县的贫困发 生率，从而得出结论：贵州旅游产业对贵州县域精准扶贫具有一定的促进作用。

关键词: 旅游产业; 贵州县域; 精准扶贫; 面板数据模型 


\section{1. 引言}

贵州社会经济发展与东部沿海发 达地区的差距, 要求贵州实行跨越式 发展, 然而, 如何实现跨越一直是贵 州省发展面临的问题。尽管具有学 习、模仿、开发的后发优势与机遇， 但同时存在基础发展条件不平等、发 展任务繁重、内生动力发展机制难以 建立等诸多制约因素。目前学术界对 如何发展这种欠发达地区特色产业主 要从文化、技术、管理等角度分析, 从经济学的角度进行定量的分析较 少。本文探索旅游产业发展对贵州县 域精准扶贫的促进作用, 为我省经济 发展提供了一定的理论与实证研究, 以弥补这方面研究的不足。

\section{2. 文献综述}

\section{1. 国内研究现状}

国内研究 “精准扶贫” 的文章和 成果目前是比较有限的, 但是也取得 了一些比较突出的研究思路。陈凤 (2014) 提到要完善扶贫对象识别机 制, 主张按照不同村不同户的特点制 定具体的帮扶规划以及措施 ${ }^{[1]}$ 。张丽杰

（2016）指出在当前我国经济新常态 的宏观背景之下, 精准扶贫工作面临 新的发展机遇, 必须认真贯彻落实习 近平总书记精准扶贫理念, 促进扶贫 理论创新、体制创新和工作机制创新 [2]。唐倩倩（2016）谈到 “精准扶贫” 作为党的十八大后重点实施的扶贫战 略新政策，是我国新时期扶贫工作机 制构建的重要思路, 但由于国土幅员 辽阔，国情复杂多样，区域差异明 显, “全国的精准扶贫成效在不同地 区会呈现不同结果 ${ }^{[3]}$; 刘海英 (2011) 主张通过扶贫机制创新向贫困对象提 供公平敦受经济社会发展新成果的机 会 ${ }^{[4]}$ 。
国内学者对于精准扶贫政策的认 识对于精准扶贫工作机制的探讨是颇 有借鉴意义的，但是对于如何有效开 展精准扶贫工作目前尚存在一些缺 失, 这些为本文的论述和思路的拓展 提供了借鉴。

\section{2. 国外研究现状}

关于贫困问题的研究, 国外学者 有着丰富的理论成果, 特别是国外关 于减贫理念的研究, 对于我国精准扶 贫理论构建和实践具有重要的实际意 义。阿玛蒂亚（2016）把产生贫困原 因归结于不平等的享受权利的现状, 权利与分配的矛盾致使出现了贫困的 现实 ${ }^{[5]}$ 。所以, 阿玛蒂亚主张解决饥荒 先要解决好不平等问题。不仅如此, 还要在生产环节、交换环节以及流通 环节给贫穷人群以基本权利, 获得平 等的机会。在阿玛蒂亚理论的指导 下，2007 年亚洲开发银行倡导以 “机 会均等” 和 “公平共享” 为要旨的减 贫理论。这一理论主张借助经济的增 长收益以及其公平分配来促进减贫效 果, 从某种程度上来说, 共享型增长 减贫理念是 “人人机会平等或人人分 享成果” 的有效减贫范式 ${ }^{[6]}$ 。

总之, 各位专家学者都表达了自 己的担忧和见解。认为政府在精准扶 贫中角色十分重要, 精准扶贫本身也 是十分复杂繁琐的工作, 想要做好这 个工作并不容易。

\section{3. 面板数据模型的建立}

\section{1. 面板数据概述}

面板数据是指在时间序列点上截 取多个面或者多个时间点, 然后把时 间序列数据和截面数据融合在一起的 所组成的二维数据集合。

面板数据被用来描述一个特定的 个体通过反复观察所形成的横截面个 
体数据和时间序列数据集, 是具有时 间、截面、个体的三维信息：从时间 序列来看，面板数据可以描述随时间 变化不同所呈现的变动规律; 从截面 数据来看，面板数据各个个体在某个 特定时间点上的个体间的差异。其表 现形式如下:

$$
\mathrm{y}_{\mathrm{it}}=\alpha_{i t}+\beta_{1 t} x_{1 i t}+\ldots+\beta_{k t} x_{k i t}+\mu_{i t},
$$$$
i=1,2, \ldots, N ; t=1,2, \ldots, T
$$

其中, $\alpha_{i t}$ 表示模型的常数项, $x=\left(x_{1 i t}, x_{2 i t}, \ldots, x_{k i t}\right)$ 表示模型中 $K$ 个 因变量, 而 $\beta_{t}=\left(\beta_{1 t}, \beta_{2 t}, \ldots, \beta_{i t}\right)$ 表示 $K$ 个变量的系数矩阵, $\mu_{i t}$ 表示随机误差 项且所满足的假设条件为: $\mu_{i t}$ 相互独 立, 且均值为 0 , 方差均为 $\sigma_{\mu}^{2}$ 。

\section{2. 模型的选择}

实证分析中我们一般通过 Hausman 检验来决定用固定效应模型还是随机 效应模型。Hausman 检验的思想是：在 $E\left(V_{i t} \mid x_{i t}\right)=0$ 的原假设下, $\hat{\beta}_{G L S}$ (广义 最小二乘估计量) 和 $\beta_{\text {Within }}$ (组内最小 二乘估计量) 都是无偏且一致的: 但在 $E\left(V_{i t} \mid x_{i t}\right) \neq 0$ 的备择假设下, $\hat{\beta}_{G L S}$ 不 仅有偏而且非一致的, $\beta_{\text {Within }}$ 是通过 组内变换消去个体效应, 因此是无偏 且一致的。

如果原假设成立, 则两种估计量 的概率极限近似, 反之则其概率极限 不同。因此 Hausman 检验则利用 $\hat{\beta}_{G L S} 、 \beta_{\text {Within }}$ 差的概率极限构造统计 量, 判断是否拒绝原假设。

\section{4. 实证分析}

\section{1. 数据来源与样本选取}

本文所有地区的年度数据全部来 自于 2014 年至 2016 年的《贵州统计 年鉴》以及各区县官方网站发布的 2014 年至 2016 年的政府工作报告和各 县政府官方网站。

（1）贫困程度指标（POV）：衡 量贫困程度的指标有贫困发生率、收 入缺口比率、阿玛蒂亚森贫困指数、 FGT 指数。本文从获得数据的便利性出 发, 采用贫困发生率来衡量贫困的程 度。

（2）旅游业发展指标（TOD）: 一个地区的旅游收入占国民生产总值 的大小无疑是反映当地旅游业发展现 状的最具说服力的指标, 故本文采用 各县旅游业年收入/GDP 来反映各县旅 游发展状况。

（3）游客吸引度指标（TOA）： 旅游业的发展必定伴随游客数量的增 加, 故本文采用各县每年接待的游客 人数来说明地区旅游业对游客的吸引 度。

(4) 农林牧渔业发展指标 (DAF) : 生态旅游渐渐成为旅游业发 展的新方向, 所以我们将农林牧渔业 的发展状况也列为一项待考察的指 标, 这里采用农林牧渔业的增加值来 表示。

（5）经济増长指标（RGP）：在 实证研究中, 人均 GDP 数据往往比 GDP 总额数据的说明性更好, 故本文采用 人均 GDP 来衡量经济的增长。

（6）收入分配指标（IG）：本文 的主体是贵州省 50 个扶贫开发重点 县, 考虑到数据的可比性和联系性, 故采用城镇人均可支配收入/农村人均 纯收入来衡量收入分配的平等情况。

基于旅游业发展对减少贫困人口 有着正向促进作用的假设，本文选取 旅游业发展水平、游客吸引度和农林 牧渔业发展水平三个因素来作为实证 模型的解释变量, 考虑到国民消费水 平对贵州县域精准扶贫的影响, 选择 
经济増长指标和收入分配指标作为模 型的控制变量。变量情况如表 4.1 所 示:

表 4.1 模型变量表

\begin{tabular}{ccc}
\hline 变量 & \multicolumn{1}{c}{$\begin{array}{c}\text { 变量 } \\
\text { 类型 } \\
\text { 名称 }\end{array}$} & 变量定义 \\
\hline 被解释 & $\mathrm{POV}$ & 贫困程度 \\
变量 & & \\
\hline 解释 & $\mathrm{TOD}$ & 旅游业发展水平 \\
\cline { 2 - 3 } 变量 & $\mathrm{TOA}$ & 游客吸引度 \\
\cline { 2 - 3 } & $\mathrm{DAF}$ & 农林牧渔业发展水平 \\
\hline 控制 & $\mathrm{RGP}$ & 经济增长 \\
\cline { 2 - 3 } 变量 & $\mathrm{IG}$ & 收入分配 \\
\hline
\end{tabular}

此外, 还有很多因素可减少贫困 发生率，例如政府部门的投资、居民 受教育的程度等, 这些影响因素我们 统一用随机误差项 $\mu_{i t}$ 来表示。得到以 下基本模型:

$$
\begin{aligned}
& P O V_{i t}=\alpha+\beta_{1} T O D_{i t}+\beta_{2} T O A_{i t} \\
& +\beta_{3} D A F_{i t}+\beta_{4} R G P_{i t}+\beta_{5} I G+\mu_{i t} \\
& \quad \text { 其中, } i=1,2,3, \ldots, 50 \text { 表示贵州省 } \\
& 50 \quad \text { 个 扶 贫 开 发 重 点 县 ; } \\
& t=2013,2014,2015 \text { 表示不同年份。 }
\end{aligned}
$$

\section{2. 旅游业对精准扶贫的影响}

\subsection{Hausman 检验}

我们认为贫困发生率会受到旅游 业发展、游客吸引度、农林牧渔业发 展、经济増长和收入分配的影响, 还 会受到其他一些与特定观察对象或时 期存在联系的因素影响。根据 2013 年 至 2015 年贵州省 50 个贫困县旅游产 业发展的原始数据, 经过简单处理, 利用 Stata11.0 软件进行 Hausman 检 验, 得到结果如表 4.2 。

表 4.2 Hausman 检验结果

\begin{tabular}{cccc}
\hline (b) & (B) & $($ b-B $)$ & $\begin{array}{c}\text { sqrt (diag } \\
\left(V_{-} b_{-}^{-}\right. \\
\text {V_B }))\end{array}$ \\
\hline fe & re & Difference & S. E.
\end{tabular}

续表 4.2 Hausman 检验结果

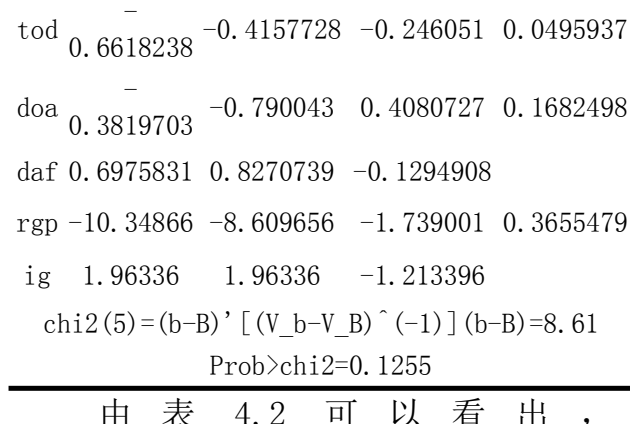
Prob $>$ chi2 $=0.1255$, Hausman 检验在 95\%的置信度下不显著, 故接受原假 设, 认为相对于运用固定效应模型进 行估计, 对样本数据运用随机效应模 型进行估计更合适。

\subsection{2 随机效应模型回归}

根据 2013 年-2015 年贵州省 50 个 国家级贫困县原始数据, 利用

\begin{tabular}{|c|c|c|c|c|c|c|}
\hline pov & Coef. & $\begin{array}{l}\text { Std. } \\
\text { Err. }\end{array}$ & $t$ & $\mathrm{P}\rangle|\mathrm{t}|$ & $\begin{array}{c}{[95 \%} \\
\text { Conf. }\end{array}$ & $\begin{array}{c}\text { Interva } \\
1]\end{array}$ \\
\hline tod & $\begin{array}{c}- \\
0.4157 \\
728\end{array}$ & $\begin{array}{c}0.1061 \\
118\end{array}$ & -3.92 & 0 & $\begin{array}{c}- \\
0.62374 \\
82\end{array}$ & $\begin{array}{c}- \\
0 . \\
20779 \\
75\end{array}$ \\
\hline doa & $\begin{array}{c}- \\
0.7900 \\
43\end{array}$ & $\begin{array}{c}0.3230 \\
725\end{array}$ & -2.45 & 0.014 & $\begin{array}{c}- \\
1.42325 \\
3\end{array}$ & $\begin{array}{c}0.15683 \\
25\end{array}$ \\
\hline daf & $\begin{array}{c}0.0270 \\
739\end{array}$ & $\begin{array}{c}0.2466 \\
582\end{array}$ & 3.35 & 0.268 & $\begin{array}{c}0.34363 \\
27\end{array}$ & $\begin{array}{c}\text { 1. } 31051 \\
5\end{array}$ \\
\hline rgp & $\begin{array}{c}- \\
\text { 8. } 6096 \\
56\end{array}$ & $\begin{array}{c}0.8618 \\
021\end{array}$ & -9.99 & 0 & $\begin{array}{c}- \\
\text { 10. } 2987 \\
6\end{array}$ & $\begin{array}{c}- \\
6.92055 \\
5\end{array}$ \\
\hline ig & $\begin{array}{c}\text { 3. } 1767 \\
56\end{array}$ & $\begin{array}{c}\text { 1. } 4763 \\
61\end{array}$ & 2.15 & 0.031 & $\begin{array}{c}0.28314 \\
11\end{array}$ & $\begin{array}{c}\text { 6. } 07037 \\
1\end{array}$ \\
\hline cons & $\begin{array}{c}30.640 \\
46\end{array}$ & $\begin{array}{c}\text { 5. } 9393 \\
59\end{array}$ & 5. 16 & 0 & $\begin{array}{c}18.9995 \\
3\end{array}$ & $\begin{array}{c}\text { 42. } 2813 \\
9\end{array}$ \\
\hline & $\begin{array}{l}\text { sign } \\
\text { sign } \\
\text { rl }\end{array}$ & $\begin{array}{l}\text { na_u } \\
\text { na_e } \\
\text { ho } \\
\text { corr }\left(u_{-}\right. \\
\quad \text { Prob }\end{array}$ & $\begin{array}{l}\mathrm{i}, \mathrm{X})= \\
>\mathrm{chi} 2\end{array}$ & $\begin{array}{c}0 \text { (assu } \\
=0.0000\end{array}$ & $\begin{array}{l}\text { 4. } 179415 \\
1.320359 \\
0.909251 \\
\text { amed) } \\
0\end{array}$ & $\begin{array}{l}56 \\
99 \\
86\end{array}$ \\
\hline
\end{tabular}
Stata11.0 软件进行参数估计, 得出如 表 4.3 所示的固定效应模型回归结 果。

表 4.3 随机效应模型回归结果 
从显著性水平来看, 旅游业发展 指标、游客吸引度指标、经济増长指 标和收入分配指标的系数显著不为 零, P 值皆小于 0.05 , 说明旅游业发 展指标和游客吸引度指标的系数为零 的可能性不到 5\%; 而农林牧渔业发展 指标 $\mathrm{P}$ 值的概率为 0.268 , 说明农林牧 渔业发展指标在 $26.8 \%$ 的概率下系数可 能为零, $73.2 \%$ 的情况下系数不为零, 所以不能通过显著性检验, 应该从模 型中删除。所以, 旅游业发展指标、 游客吸引度指标、经济増长指标和收 入分配指标均对贵州 50 个贫困县的贫 困发生率存在显著影响，其弹性分别 为 $0.4158 、 0.79 、 8.6097 、 3.1768$, 而农林牧渔业发展指标则不存在显著 影响。

\section{2.3 删除不显著项后 Hausman 检验}

将原始数据中删除收入分配 （IG）这一控制变量，利用 Stata11.0 软件进行 Hausman 检验, 结果见表 4. 4 。

表 4.4 Hausman 检验结果

\begin{tabular}{|c|c|c|c|}
\hline (b) & (B) & $(b-B)$ & $\begin{array}{l}\text { sqrt (diag } \\
\left.\left(V_{-} b-V_{-} B\right)\right)\end{array}$ \\
\hline fe & re & $\begin{array}{c}\text { Differen } \\
\text { ce }\end{array}$ & S. E. \\
\hline- & - & - & \\
\hline tod 0.629658 & 0.406784 & 0.222847 & 0.0547451 \\
\hline 4 & 3 & 1 & \\
\hline $\begin{array}{c}- \\
\text { doa } 0.266647\end{array}$ & $\begin{array}{c}- \\
0.746052\end{array}$ & $\begin{array}{c}0.479404 \\
9\end{array}$ & 0.1825376 \\
\hline 2 & 2 & & \\
\hline $\begin{array}{c}\text { rgp } \\
\text { 11. } 43676\end{array}$ & $\begin{array}{c}- \\
9.641491\end{array}$ & $\begin{array}{c}- \\
1.795274\end{array}$ & 0.3757357 \\
\hline $\begin{array}{l}\text { ig } 2.748999 \\
\operatorname{chi2}(4)=(b-\end{array}$ & $\begin{array}{l}\text { 4. } 232755 \\
-B)^{\prime}\left[\left(V_{-} b^{-}-\right.\right.\end{array}$ & $\begin{array}{c}- \\
\text { 1. } 483756 \\
\left.\left.-V_{-} \text {B }\right)^{\wedge}(-1)\right]\end{array}$ & $(b-B)=3.84$ \\
\hline \multicolumn{4}{|c|}{ Prob $>_{\text {chi } 2=0.4279}$} \\
\hline
\end{tabular}

由表 4.3 可以看 出, Prob $>$ chi $2=0.4279$, Hausman 检验在 95\%的置信度下不显著, 故接受原假 设, 认为相对于运用固定效应模型进
行估计, 对样本数据运用随机效应模 型进行估计更合适。

\subsection{4 随机效应模型回归}

根据 2013 年至 2015 年贵州省 50 个国家级贫困县原始数据, 删除农林 牧渔业发展指标后, 再次利用 Stata11.0 软件进行参数估计, 得出如 表 4.5 所示的随机效应模型回归结 果。

表 4.5 随机效应模型回归结果

\begin{tabular}{|c|c|c|c|c|c|}
\hline pov & Coef. & $\begin{array}{l}\text { Std. } \\
\text { Err. }\end{array}$ & $\mathrm{t} \quad \mathrm{P}>|\mathrm{t}|$ & $\begin{array}{c}{[95 \%} \\
\text { Conf. }\end{array}$ & $\begin{array}{c}\text { Interva } \\
1]\end{array}$ \\
\hline tod & $\begin{array}{c}- \\
0.40678 \\
43\end{array}$ & $\begin{array}{c}0.1095 \\
295\end{array}$ & $-3.71 \quad 0$ & $\begin{array}{c}- \\
0.6214 \\
581\end{array}$ & $\begin{array}{c}- \\
0.19211 \\
05\end{array}$ \\
\hline doa & $\begin{array}{c}- \\
0.74605 \\
22\end{array}$ & $\begin{array}{c}0.3336 \\
913\end{array}$ & -2.240 .025 & $\begin{array}{c}- \\
\text { 1. } \\
4000 \\
75\end{array}$ & $\begin{array}{c}0.09202 \\
93\end{array}$ \\
\hline rgp & $\begin{array}{c}- \\
\text { 9. } 64149 \\
1\end{array}$ & $\begin{array}{c}0.8373 \\
706\end{array}$ & $\begin{array}{cc}- \\
11.51\end{array}$ & $\begin{array}{c}- \\
11.282 \\
71\end{array}$ & $\begin{array}{c}- \\
\text { 8. } 00027 \\
4\end{array}$ \\
\hline ig & $\begin{array}{c}\text { 4. } 23275 \\
5\end{array}$ & $\begin{array}{c}1.4779 \\
59\end{array}$ & 2.680 .004 & $\begin{array}{c}\text { 1. } 3360 \\
09\end{array}$ & $\begin{array}{c}\text { 7. } 12950 \\
1\end{array}$ \\
\hline $\begin{array}{c}-\mathrm{co} \\
\mathrm{ns} \\
\text { sig }\end{array}$ & $\begin{array}{c}\text { 34. } 8064 \\
1\end{array}$ & $\begin{array}{c}5.9912 \\
94\end{array}$ & $5.81 \quad 0$ & $\begin{array}{c}23.063 \\
69\end{array}$ & $\begin{array}{c}46.5491 \\
4\end{array}$ \\
\hline $\mathrm{ma}_{-}$ & & & 4. 4968712 & & \\
\hline $\mathrm{ma}_{-}$ & & & 1. 3852328 & & \\
\hline rho & & $\begin{array}{r}\operatorname{corr}\left(u_{-}\right. \\
\text {Pro }\end{array}$ & $\begin{array}{c}0.913333 \\
\mathrm{i}, \mathrm{X})=0 \text { (assu } \\
>\text { chi2 }=0.0000\end{array}$ & med) & \\
\hline
\end{tabular}

由表 4.5 可知, 从变量的估计系 数来看, 旅游业发展指标、游客吸引 度指标、经济増长指标与贵州贫困县 的贫困发生率呈负相关关系，说明旅 游业发展、游客吸引度和经济増长的 增加可以减缓贵州国家级贫困县的贫 困现状, 收入分配与贫困发生率呈正 相关关系, 即收入分配差距越大, 贵 州省国家级贫困县的贫困发生率越 高, 这与现实经济意义相符。从变量 的估计系数来看, 旅游业发展指标、 
游客吸引度指标、经济増长指标和收 入分配指标的 P 值均小于 0.05 , 所以 其在 95\%置信度下均显著。所以, 解释 变量中的旅游业发展指标、游客吸引 度指标对减少贵州贫困县的贫困发生 率有积极作用, 由此可以得到回归方 程:

$$
\begin{aligned}
& P V_{i t}=34.8064-0.4068 T O D_{i t}-0.7461 T O A_{i t} \\
& -9.6415 R G P_{i t}+4.2328 I G+\mu_{i t}
\end{aligned}
$$

\section{5. 结束语}

运用随机效应模型进行实证研究 发现, 旅游业发展、游客吸引度和经 济増长的增加可以减缓贵州国家级贫 困县的贫困现状，收入分配差距越 大，贵州省国家级贫困县的贫困发生 率越高。因此, 除了增加旅游业投入 以促进旅游产业的发展外, 提升旅游 景区知名度或发展旅游经济的规模也 是促进减少贫困人口的一个重要的途 径。从显著性水平来看, 对贵州贫困 发生率存在显著影响因素的是旅游业 发展和经济増长, 旅游人口显著性水 平稍低, 可能是由于贵州省旅游消费 水平参差不齐造成的。

通过建立面板数据模型研究表 明, 加大各地区旅游产业投资对降低 贫困发生率有一定的积极作用。投资 不仅仅只是扶贫办公室政府加大投 资，同时可以鼓励企业家对贫困地区 产业帮扶，提供资金或者技术实现双 赢。政府加大财政支出投入, 加大对 公共基础设施建设, 有效缓解偏远地 区道路交通问题。

近年来学者们对于贫困问题的研 究不少, 但是关于贫困发生率的研究 并不多见。本文以旅游产业为切入 点, 收集贵州省 50 个县的面板数据, 通过对数据进行简单处理后建立面板 数据模型, 比较优劣, 从而确定最终 合适模型, 并得出结论。从研究内 容、数据的收集及模型建立实证分析
具有一定创新性。但由于本人的能力 有限, 在研究分析的深度、变量选择 方法及深度上较为粗浅, 所以本文在 数据扩展方面及影响因素的拓展仍有 一定的研究空间。

\section{致谢}

本文为贵州财经大学在校学生科 学研究项目《贵州特色产业扶贫的金 融支持效率及提升机制研究》的阶段 性成果之一。

\section{参考文献}

[1] 陈风. 贵州省贫困发生率面板数据 统计分析 [D]. 贵州财经大学, 2014.

[2] 张丽杰. 县级政府精准扶贫政策执 行问题研究 [D]. 郑州大学, 2016 .

[3] 唐倩倩. 金融支持战略性新兴产业 发展的内在机理及效率评价研究 [D]．贵州财经大学，2016.

[4] 刘海英. 大扶贫: 公益组织的实践 与建议 $[\mathrm{M}]$. 社会科学文献出版社, 2011.

[5] He X. Poverty Alleviation in Zang People's Regions of Yunnan Province from the Perspective of Accurate Antipoverty $[\mathrm{C}] / /$ International Conference on Society Science. 2017.

[6] Yang Y, Zhang A. Precise Poverty Alleviation Problems and Countermeasures in Xinjiang Uygur Autonomous Region $[\mathrm{C}] / / \quad$ International Education, Economics, Social Science, Arts, Sports and Management Engineering Conference. 2016. 